\title{
Clarifying a crowded field of techniques
}

\author{
Tissue clearing methods let researchers see inside their animal models. However, there are a lot of options out \\ there. Which to choose can depend on your question.
}

\section{Michael Eisenstein}

$f y$ f you find yourself traveling via Tokyo's Haneda Airport, you may pass through a gallery with a striking photograph of a crystal-clear brain. Although it has the appearance of glass, this is in fact a genuine mouse brain rendered completely transparent through a technique developed by Hiroki Ueda's team at the RIKEN Center for Biosystems Dynamics Research in Osaka, Japan. Ueda's method, known as CUBIC $^{1}$ - short for 'clear, unobstructed brain imaging cocktails and computational analysis' - is just one of a myriad of methods that have emerged over the past 15 years for 'tissue clearing' in a wide range of organs.

These cleared specimens are giving researchers a literal window into the structure, organization, and function of intact tissues. "We have now created a single-cell, three-dimensional mouse brain atlas," says Ueda, whose lab is using brain imaging to address questions such as how the central nervous system establishes and maintains the internal day-night clock known as the circadian rhythm. In the past, this kind of research would require the laborious collection and imaging of ultrathin two-dimensional tissue slices, which must then be painstakingly computationally reconstructed into a three-dimensional volume.

Even after all that work, sectioning-based methods may fall short for some experiments. For example, Alan Watson of the University of Pittsburgh had long struggled in his efforts to study patterns of encephalitic virus infection in the mouse brain. "We were essentially looking for what could potentially be just one or two cells in an entire brain, and you can imagine pretty quickly that that becomes near-impossible," says Watson. But by applying tissue-clearing, his team was suddenly able to comb through the brain in its entirety and home in on the relevant cell populations ${ }^{2}$. One can even clear an entire mouse body, enabling researchers to home in on ultra-rare cells such as tumor metastases or reservoirs of stem cells ${ }^{3}$.

These techniques are powerful, and in many cases relatively straightforward to

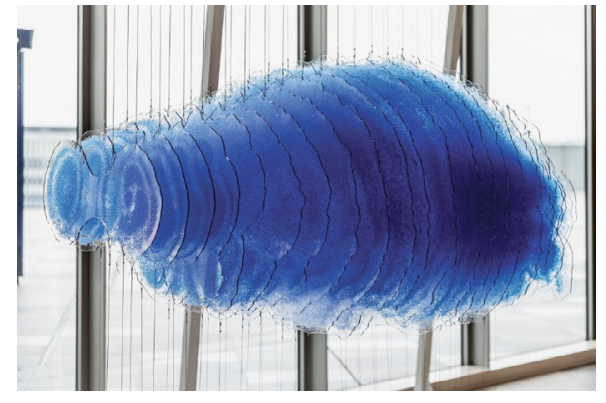

An acrylic brain on display at Tokyo's Haneda Airport. Credit: RIKEN Center for Biosystems Dynamics Research

perform. But planning an experiment can nevertheless be a fraught decision because of the large and ever-growing number of tissue-clearing methods in the literature. And each of these has its own advantages and limitations, which may make it a better or worse fit for a particular research question. "The mistake that I think most people make is that they assume that "clearing is clearing," says Watson, "and they just go for it without considering the implications of the experimental context."

\section{A problem with many solutions}

Our bodies are opaque because of the light-scattering properties of the molecules contained within our cells. These include the lipids found in biological membranes, and a variety of pigments such as the heme that produces the distinctive red color of blood. Tissue-clearing protocols work by removing these light-reflecting compounds while still largely preserving the underlying composition of the tissue. These techniques can be broken down into three major categories: solvent-based, hydrophilic, and hydrogel-based.

Solvent-based techniques employ a series of sample-immersion steps. The specimen is first dehydrated, eliminating the light-scattering effects of water molecules. Subsequent treatments remove lipids from the tissue, which is then transferred to a medium with a similar refractive index- a physical property describing how the angle of light changes upon entering a particular substance. If the tissue's refractive index matches that of the surrounding medium, it becomes effectively transparent. These were the first generation of clearing methodsindeed, the earliest example of solvent-based clearing dates back to the 1914 work of German physiologist Werner Spalteholz. However, this method largely failed to take off due to the limitations of available imaging technologies.

Over the past few decades, other researchers have developed contemporary adaptations of the Spalteholz technique, but perhaps the most influential of these is ' $3 \mathrm{D}$ imaging of solvent-cleared organs' (3DISCO), developed by a group led by Hans-Ulrich Dodt at the Technical University of Vienna in Austria in 2012. Initially, Dodt's team struggled to find a formula that could clear tissue without also wiping out signal from reporters like green fluorescent protein (GFP) a potential deal breaker for studies that rely on these indicators to track gene expression. "You had to image very quickly-within six hours," recalls Dodt. "I had a Master's student who basically sat beside the brain, and once it got cleared, he rushed it under the microscope." Subsequent iterations of 3DISCO overcame this problem to some extent, while still producing astonishingly transparent tissues within the space of a day or two. A host of specialized 'DISCO'-family techniques have since proliferated, such as the iDISCO method $^{5}$ developed in Marc Tessier-Lavigne's lab at Rockefeller University, which is optimized for imaging with labeled antibodies.

Hydrophilic methods, such as Ueda's CUBIC, initially emerged as a gentler alternative to 3DISCO. "At that time, there was no safe and also easy to use method that preserves fluorescence and also is high performance," says Ueda. "They are also relatively unsafe, because you need to protect yourself from the solvent." The initial CUBIC method, published in $2014^{1}$, employs chemicals known as aminoalcohols, which eliminate lipids from the tissue while 


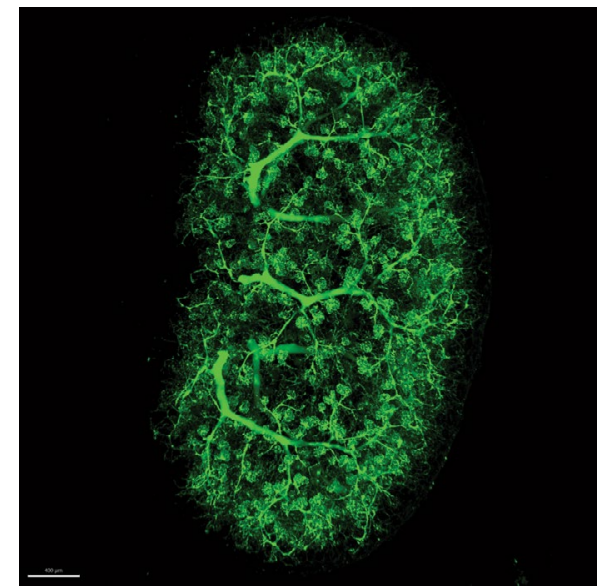

Vasculature within a neonatal mouse kidney cleared using CUBIC. Credit: Alan Watson,

Jacqueline Ho and Sunder Sims-Lucas, University of Pittsburgh

also promoting water uptake, such that the specimen becomes clear in solution. In subsequent work, his team conducted extensive screening of a 1,600-chemical library to develop a more optimized CUBIC protocol $^{6}$ that achieves even greater transparency in a wider range of organs and tissues.

Around the same time, Karl Deisseroth's group at Stanford University pioneered the development of hydrogel-based techniques with a method known as CLARITY ${ }^{7}$.

CLARITY involves soaking a tissue in a mild detergent, which breaks down cellular lipids into tiny bubbles known as micelles. The sample is then infused with a solution of molecules that can be triggered to self-assemble into a stable hydrogel scaffold, which also binds to the various proteins present in the tissue and locks them into place. Finally, an electric current is applied to the tissue, driving out the lipid micelles while leaving the transparent, protein-coupled hydrogel behind.

Although effective, this approach can be tough on tissues. "It's not good if you want to look at sensitive or sparse epitopes... it can be destructive," says Jennifer Treweek, of the University of Southern California. As a postdoc for Viviana Gradinaru, who initially helped develop CLARITY in Deisseroth's lab, Treweek devised gentler alternatives to this technique ${ }^{8}$, in which the clearing and hydrogel solutions are directly perfused into animals, and no electrical current is required. "It's basically simplifying the formulation and preparation of the tissue a bit," says Treweek, "with the idea that if you want to look at sensitive epitopes, you need to have perfect preservation of the information content in tissue."
Matching the method to the model

But this brief tour of techniques is only scratching the surface-as of late 2020, roughly 50 different clearing protocols had been published in the literature, and the field shows no sign of slowing down. "There are too many methods available now," says Dan Zhu, of the Huazhong University of Science and Technology in China, noting that researchers who are not already familiar with tissue clearing "may not be able to choose a proper method for their experiments, leading to unsatisfactory results." She and other experts have published recent reviews (ex. Refs. ${ }^{9,10}$ ) that provide head-to-head comparisons of various techniques, but there are also experimental considerations that can help scientists to narrow down their choices.

One of the most important factors is the labeling strategy being used. For researchers working with genetically encoded fluorescent proteins such as GFP or its various multi-colored cousins, hydrophilic methods are probably the best option in terms of speed, ease-of-use, and preservation of signal. "Generally, the first thing we'll try is CUBIC," says Watson, who routinely develops clearing protocols for collaborators at his university's Center for Biologic Imaging. Even though things have improved since the early days of DISCO, solvent-based methods still have a tendency to quash fluorescence signal, but some newer techniques offer greatly improved performance. For example, Dodt's team has identified 'antifading' reagents that can preserve signal from certain fluorophores ${ }^{11}$, and Zhu and colleagues have shown that subtle tweaks to the temperature and $\mathrm{pH}$ of the 3DISCO reagents can greatly improve long-term signal stability ${ }^{12}$. "Years later, the fluorescence signal can be preserved very well," says Zhu. However, not all fluorophores will benefit from these adjustments.

On the other hand, iDISCO ${ }^{5}$ is probably the best starting point for experiments involving antibody-based labeling. "We developed a solvent gradient to break down all of the cellular membrane structures to allow the antibody to go in to label the entire structure," says Zhuhao Wu of the Icahn School of Medicine at Mount Sinai, who co-developed the method as a postdoc in Tessier-Lavigne's lab. "We also figured out how to make sure that the antibody would penetrate well by blocking various 'sticky' parts of the tissue." Treweek notes that the iDISCO team went to great lengths to detail their protocol and validate a wide range of antibodies for labeling. "I think almost every tissue-clearing protocol still falls back on using iDISCO immune-labeling procedures, even if they don't use the actual clearing procedure," she says.

Hydrogel techniques are compatible with both labeling methods, but are generally slower or more expensive than other methods-indeed, the original CLARITY method requires the use of costly, specialized equipment. But these are also the only clearing methods that can currently be used for the detection of RNA targets as well as proteins, whereas hydrophilic and solvent-based methods tend to degrade

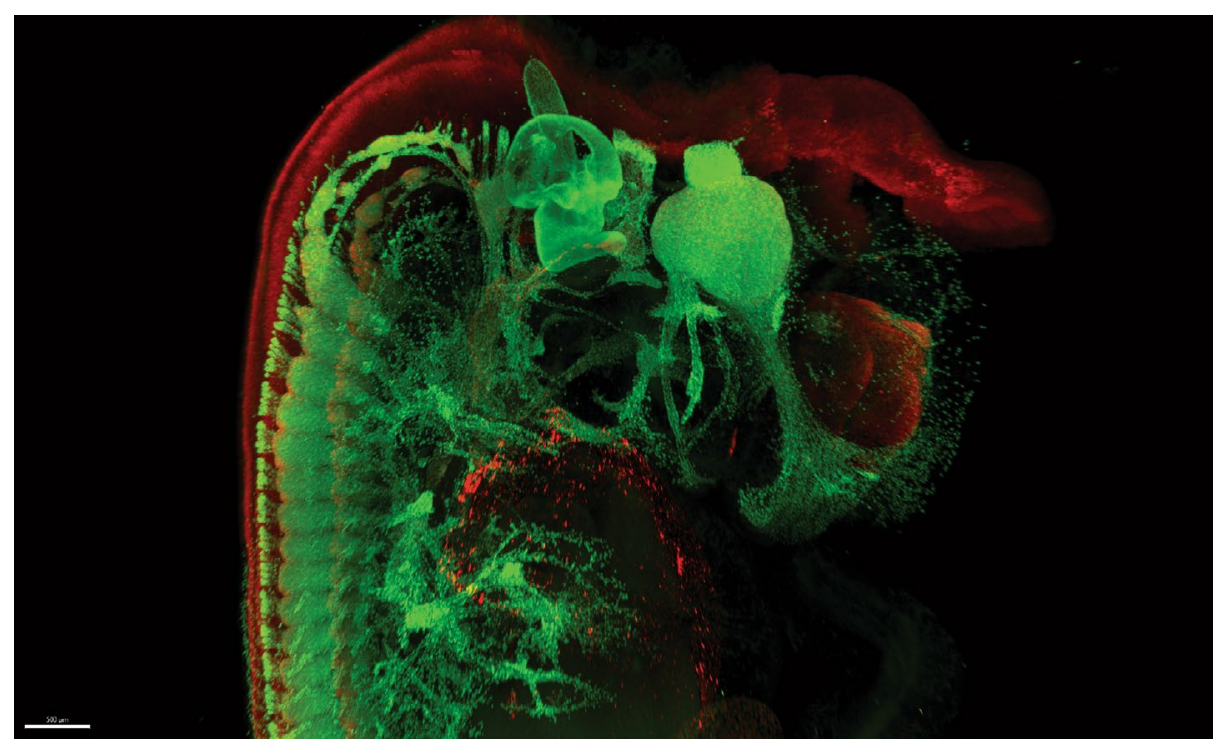

A 12.5 day mouse embryo cleared using CUBIC. The peripheral nervous system is labeled for Sox10 (green) with developing neurons labeled in red. Credit: Alan Watson, University of Pittsburgh and Michelle Southard-Smith, Vanderbilt University. 


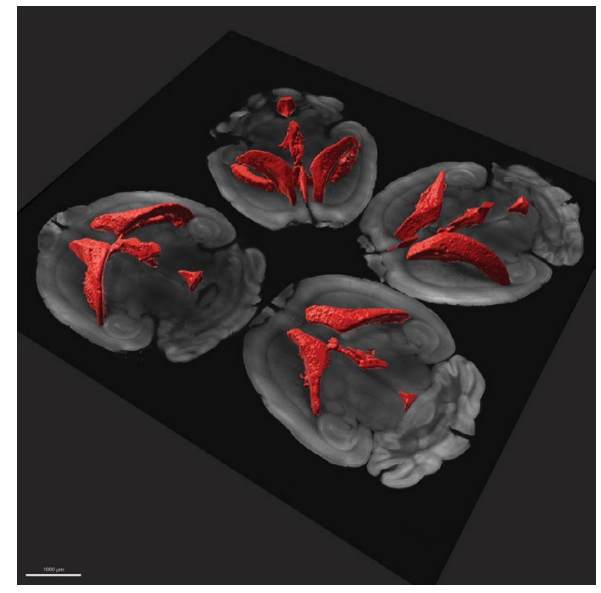

Brains from two normal and two irradiated mice cleared using 3DISCO and imaged by reflected light confocal. Surface rending of ventricles (red). Credit: Alan Watson, Simon Watkins, Michael Epperly, and Joel Greenberger, University of Pittsburgh.

these molecules. "Hydrogel embedding allows you to stabilize those nucleic acids," says Treweek, adding that this allows you to obtain spatial information about where various genes are expressed within a tissue that would be inaccessible to other RNA analysis techniques.

The target organ also matters. These techniques have generally been well-refined for use in the brain and can often be adapted to a range of other organs with minor adjustments. However, some experiments pose additional challenges that require more specialized clearing protocols. For example, Ueda says that in the course of screening their chemical library for CUBIC optimization $^{6}$, they identified chemical combos that are particularly well-suited for extracting the minerals from bone or clearing out pigments like melanin from the skin or eye. Watson reports that a few particular tissues remain stubbornly difficult, including muscle and liver, and he has generally found conventional CLARITY to be the best strategy for clearing these. "It's probably just the horsepower-we have some electrical current behind it really forcing all those lipids and extra molecules out of the tissue," he says.

Finally, one must take into account the impact that tissue-clearing procedures have on the tissue itself. Given that the solvent-based methods entail a dehydration step, cleared tissues will generally shrink to some extent-by several-fold in some protocols. This can be an advantage in terms of making large specimens more manageable for microscopy, as in experiments where researchers aim to clear entire animals. But it can also undermine the resolution of more targeted studies, making it even harder to discriminate individual cells and protein signals in tissues with high cellular density. "It also concentrates all the other signals that you aren't necessarily interested in, like the autofluorescence background that you're trying to overcome during imaging," says Watson. Conversely, hydrogel and hydrophilic methods can cause tissues to swell; the resulting expansion may be a boon in terms of being able to resolve greater detail, but researchers must be mindful of the potential for artifacts. "If you have a hydrogel, that sort of stabilizes the tissue and ensures that any swelling is even," says Treweek, whereas some hydrophilically cleared tissues may be more prone to irregular swelling or puckering as they expand.

\section{Getting a clearer picture}

Given the complexities associated with planning the ideal clearing strategy for a given experiment, many experienced users opt for a 'buffet-style' experimental design that combines the best features of multiple methods. "Very rarely do we actually use just CUBIC or just DISCO," says Watson, noting that he increasingly finds himself using the hydrogel components from CLARITY to reinforce tissues cleared with CUBIC. And Zhu's team has been working on a hybrid strategy that pairs aspects of their solvent-based 'fDISCO' technique ${ }^{12}$ with a hydrophilic technique developed in their lab. "The new method makes tissues that are very, very clear," she says.

Regardless of the method selected, all clearing experiments tend to converge upon a similar set of challenges on the imaging and analysis side. Light-sheet microscopy is the imaging platform of choice for virtually all tissue-clearing experiments. Rather than scanning with a focused laser as in a confocal microscope, these instruments illuminate samples with a two-dimensional sheet of light, enabling the rapid collection of image data from relatively large volumes. "Light-sheet can image clarified tissues much faster compared to confocal, with lower photobleaching," says Peng Fei, a collaborator of Zhu's at the Huazhong University of Science and Technology. In one recent publication ${ }^{13}$, their team described an imaging strategy that allowed them to achieve high-quality volumetric imaging of an entire mouse brain within a matter of minutes.

But light-sheet experiments can also be tricky to set up-particularly if one is looking to image specimens larger than an isolated mouse organ. "They're beautiful systems, but they have to be designed very specifically for the type of clearing and the type of tissues that you're trying to image," says Watson. Several groups have demonstrated the feasibility of using existing clearing techniques on organs from bigger animal models - for example, Ueda's team has used CUBIC to render a hemisphere of the marmoset brain transparent ${ }^{14}$. However, imaging these tissues is no mean feat. It requires an appropriately designed instrument and ample time, and light-sheet image quality falters as one looks deeper and deeper into a thick specimen. "Even with an entire mouse brain, we still observe degradation of image quality," says Fei.

These experiments can also leave researchers drowning in data. "You're probably producing a bare minimum of about a terabyte of data per day, and that's per fluorescence channel," says Watson. A few groups have developed software that can help wrangle these tremendous volumes of data. For example, the iDISCO team devised an analytical tool called ClearMAP ${ }^{5}$ that is now widely used in the field. Even with a robust algorithmic pipeline, the regular output of a clearing project can tax a lab's computational capacity, and Fei says that his team relies on high-performance computing workstations to manage the data load.

But even with these challenges, tissue clearing has already proven to be a transformative tool for scientists at every level. Ueda notes that the CUBIC reagentswhich are now commercially available-are already being used as a learning tool in some high school science labs. In the meantime, his team is already making plans for their next ambitious project. "I want to make every single organism into a digitalized version-so now we are trying to digitalize a rat brain and a marmoset brain," says Ueda.

\section{Michael Eisenstein ${ }^{凶}$}

Freelance science writer, Philadelphia, PA, USA.

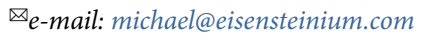

Published online: 16 June 2021

https://doi.org/10.1038/s41684-021-00796-x

\footnotetext{
References

1. Susaki, E. A. et al. Cell 157, P726-P739 (2014)

2. Watson, A. M. et al. PLoS One 12, e0180486 (2017).

3. Kubota, S. I. et al. Cell Rep 20, 236-250 (2017).

4. Becker, K. et al. PLoS One 7, e33916 (2012).

5. Renier, N. et al. Cell 159, P896-910 (2014).

6. Tainaka, K. et al. Cell Rep 24, P2196-2210.E9 (2018).

7. Chung, K. et al. Nature 497, 332-337 (2013).

8. Yang, B. et al. Cell 158, P945-958 (2014).

9. Wan, P. et al. Neurophotonics 5, 035007 (2018).

10. Xu, J. J Biophotonics 12, e201800134 (2019).

11. Hahn, C. et al. J Biophotonics 12, e201800368 (2018).

12. Qi, Y. et al. Sci $A d v$ 5, eaau8355 (2019).

13. Fang, C. et al. Nat Commun 12, 107 (2021).

14. Susaki, E. A. et al. Nat Commun 11, 1982 (2020).
} 